File 2650 ja-1 2nd print (3)

Bangladesh J. Sci. Ind. Res. 42(3), 257-268, 2007

\title{
A New DC Model for Transmission Loss Allocations in Power Systems
}

\author{
M. H. Kabir \\ Department of Computer Science and Telecommunication Engineering, Noakhali Science \\ and Technology University, Sonapur, Noakhali 3802. Bangladesh
}

\begin{abstract}
Under open access environment in the era of deregulated power industry, transmission loss allocation to various transactions is one of the important issues to be solved exactly. Because of the non-linear nature of power flow and power loss, it is very difficult to segregate and to allocate losses among the participants properly. This paper presents a New DC method (NDC) where an efficient loss allocation algorithm has been applied. Considering the impact of every line flow in a network, the loss allocations to the loads have been computed at first and finally the total loss has been allocated to the generators. The effectiveness of this procedure has been studied for the 6-bus and IEEE 118-bus model power systems. Several numerical analyses implicate that the proposed loss allocation procedure including the DC load flow model is reasonably acceptable.
\end{abstract}

Keywords: Transmission loss allocation DC load flow model, Deregulated power market

\section{Introduction}

The market driven transactions, under open access environment, have become the new independent decision variables defining the behavior of the power system. Transmission losses form a significant component of the amount of power that has to be generated in order to meet the power demand. As an example, in a power network with a demand of 10,000 MW and $7 \%$ transmission losses, the implication is that the generation must be capable of supplying $10700 \mathrm{MW}$, an extra $700 \mathrm{MW}$, fully a large power plant that must be built and operated. Clearly, someone must pay for both the capital investment and the fuel needed to generate the $700 \mathrm{MW}$ of lost power. In the traditional utility, this cost is bundled into the rates together with other ancillary services and charged in some prorata fashion (Kirschen, et al. 1997; Conejo, et al., 2001; Gross and Tao, 2000; Conejo et al., 2002). With competition, this practice still persists but, more and more, there will be a need to allocate losses to transactions in a more systematic manner, particularly one that will account for the network location of the buyer and seller as well as the non-linear 
interaction among simultaneous transactions. For example, transactions where the seller and buyer are electrically close may not generate much in the way of losses. Similarly, some transactions may actually reduce overall system losses while others can have an opposite effect. Methods that can systematically identify such differences in behavior are therefore required. There is no unique or ideal procedure existed for loss allocation, but they should have most of the desirable properties stated below.

a. To be consistent with the result of a power flow;

b. To depend on the amount of energy either produced or consumed;

c. To depend on the relative location in the transmission network;

d. To avoid volatility;

e. To provide appropriate economic marginal signals;

f. To be easy to understand;

g. To be simple to implement.

Our procedure shows almost all of the properties stated above. The main feature of our procedure is that the real impact of power flow in every transmission line has been considered extensively and properly by developing loss coefficient matrices (one for loss allocations to loads and the other for loss allocations to the generators). This procedure allocates losses to the loads at first and then to the generators. To develop the proposed method, we only need to know the power injections in the generator and load demand buses along with the network parameters. As a result, the procedure is easy to understand, very simple to implement and very fast in computations of total loss and its allocations to loads and to generators. The suggested procedure takes care for the geographical position of the buses.

In a similar way to incremental loss allocation methods Galiana, et al., 2002; Galiana, et al., 2003; Mateus and Franco 2005), this procedure can yield negative loss allocation to reward geographically well-positioned generators or loads in the network (Conejo, et al., 2001).

\section{Mathematical Formulation}

From the known power injections at the generator (PV) buses a matrix $\boldsymbol{f}$ has been developed that establishes a prompt relationship between the power injections at PV buses to the injections at the load demand (PQ) buses as shown below.

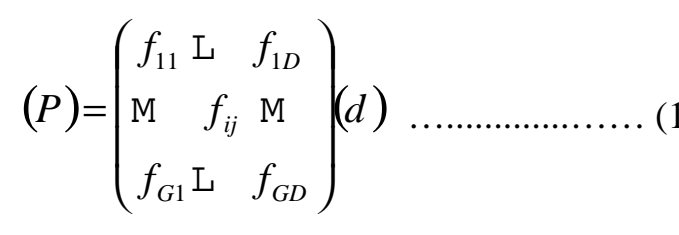

Where $\boldsymbol{P}$ consists of net power injections in the PV buses, $\boldsymbol{d}$ consists of gross power injec- 
tions (including losses) at the PQ buses. At first, taking penalty factor $=1$ for all the generating units, the power outputs of the generators are determined by Economic Load Dispatch (ELD) mode of operation. In other cases the net power injections in the PV buses are calculated by Eq.1. As a result, this procedure neither depends on Incremental Transmission Loss (ITL) of buses nor on arbitrary slack bus selection. Hence, it is a non-volatile procedure. The elements $f_{11}, \ldots$, $f_{\mathrm{G} 1}$ are calculated as follows

$f_{i 1}=\frac{\text { Net power injection at PV bus i }}{\text { Sum of net power injections at PV buses }} .$.

Expressing the $\boldsymbol{f}$ as Eq. 3.

$$
(F)=\left(\begin{array}{ccc}
f_{11} \mathrm{~L} & f_{1 D} \\
\mathrm{M} & f_{i j} & \mathrm{M} \\
f_{G 1} \mathrm{~L} & f_{G D} \\
-1 & & 0 \\
& 0 & \\
0 & & -1
\end{array}\right) \ldots \ldots \ldots \ldots \ldots \ldots \ldots
$$

The power injection matrix $\boldsymbol{I}$ at all buses can be expressed as follows:

$$
(I)=(F)(d)=(B)(\delta)
$$

Where $\boldsymbol{F}$ is an $(n \times D)$ matrix, $\boldsymbol{B}$ is an $(n \times n)$ susceptance matrix, $\delta$ is an $(n \times 1)$ column vector of node voltage phase angles. Here, $n=$ total bus number, $D=$ number of PQ buses. Therefore, from Eq. 4 the matrix $\delta$ can be expressed as

$$
(\delta)=\left(B^{-1}\right)(F)(d)
$$

In DC load flow method (Exposito, et al., 2000; Wood and Wollenberg, 1996; Yamashiro, 1977 and Conejo, et al. 2003), a power flow matrix $P_{F}$ for the whole system can be written as follows.

$$
\begin{aligned}
\left(\mathrm{P}_{F}\right) & =(b)(A)(\delta) \\
& =(b)(A)\left(B^{-1}\right)(F)(d) \\
& =(\mathrm{K})(\mathrm{d})
\end{aligned}
$$

Where $\mathbf{b}$ is an $(m \times m)$ diagonal matrix that consists of transmission line susceptances as diagonal elements. $\boldsymbol{A}$ is an $(m \times n)$ matrix that contains 1 for starting nodes and -1 for ending nodes and rest of the elements is zero, $\boldsymbol{K}$ is an $(m \times D)$ matrix, $m=$ number of transmission lines. Therefore, power flow in line $l$ can be written as

$$
\left(\mathrm{P}_{F 1}\right)=\left(K_{1}\right)(d)
$$

Where, $\boldsymbol{K}_{\boldsymbol{l}}$ is the $\boldsymbol{l}^{\text {th }}$ row of the matrix $\boldsymbol{K}$. In DC method, power flow in a line is assumed to be current. Therefore, power loss in the line $l$ is computed as

$$
\begin{aligned}
L_{l} & =\left(P_{F l}\right)^{2} r_{l}=\left(K_{l} d\right)^{2} r_{l} \\
& =\left(d^{T}\right)\left(K_{l}^{T}\right)\left(K_{l}\right)(d) r_{l} \\
& =\left(d^{T}\right)\left(S^{l}\right)(d)=\sum_{i=1}^{D} \sum_{q=1}^{D} d_{i} S_{i q}^{l} d_{q} \\
& =\sum_{i=1}^{D} d_{i}^{2} S_{i i}^{l}+\sum_{i=1}^{D} \sum_{\substack{q=1 \\
q \neq i}}^{D} d_{i} S_{i q}^{l} d_{q}
\end{aligned}
$$

Where $r_{l}$ is the resistance of the transmission line $l$. The coefficient $\left(S^{1}\right)=\left(K_{l}^{\mathrm{T}}\right)\left(K_{l}\right) r_{l}$ is a 
square matrix developed to allocate the loss of line $I$ to the PQ buses. Because of this loss coefficient matrix, the procedure accounts for the geographical location of the PQ buses in the network.

Assuming that the loss caused by $d_{i}$ and $d_{q}$ is equally allocated to each node of $i$ and $q$ (Exposito, et al. 2000). From Eq.8 the allocated loss $L_{D l i}$ to PQ bus $i$ for transmission line $l$ can be written as follows:

$$
L_{D l i}=d_{i}^{2} S_{i i}^{1}+\sum_{\substack{\mathrm{q}=1 \\ \mathrm{q} \neq 1}}^{\mathrm{D}} d_{i} S_{i q}^{1} d_{q}
$$

Similarly, loss allocations considering the impact of all other line flows in the network can easily be computed. Therefore, total loss allocated to PQ bus $i$ is as

$$
L_{D i}=\sum_{l=1}^{\mathrm{m}} L_{D l i}
$$

The sum of the allocated losses to PQ buses is exactly equals to the sum of the losses in transmission lines as calculated by using Eq.8. Adding the allocated losses to the respective loads, the elements in $\boldsymbol{d}$ are refreshed and used in Eq.1. In the proposed method, the Eq.1 through Eq.10 are repeated until the network converges. It is worth noting that the proposed method starts from the no loss condition.

Finally, to allocate the transmission losses to the PV buses, the following mathematical formulation has been proposed. Now, Eq.11 shows the relationship between the power injections at PQ buses to the injections at the PV buses.

$$
(d)=\left(\begin{array}{lll}
c_{11} \mathrm{~L} & c_{1 G} \\
\mathrm{M} & c_{i j} & \mathrm{M} \\
c_{D 1} \mathrm{~L} & c_{D G}
\end{array}\right)(P)
$$

The elements $c_{11}, \cdots, c_{D 1}$ are calculated from the elements in $\mathbf{d}$ that is exactly as follows:

$\mathrm{C}_{i 1}=-\left(\frac{\text { Load }+ \text { allocated loss to load bus } \mathrm{i}}{\text { Total load }+ \text { Total loss }}\right)$

Expressing the $c$ as Eq. 3.

$$
(C)=\left(\begin{array}{lll}
1 & & 0 \\
& 0 & \\
0 & & 1 \\
c_{11} \mathrm{~L} & c_{1 G} \\
\mathrm{M} & c_{i j} & \mathrm{M} \\
C_{D 1} \mathrm{~L} & c_{D G}
\end{array}\right)
$$

The power injection matrix $\boldsymbol{I}$ at all buses can be expressed as follows:

$$
(l)=(C)(P)=(B)(\delta)
$$

Therefore, the power flow matrix $\boldsymbol{P}_{\boldsymbol{F}}$ in Eq.6 for the whole system can be expressed as follows:

$$
\begin{aligned}
\left(P_{F}\right) & =(b)(A)\left(B^{-1}\right)(C)(P) \\
& =(H)(P)
\end{aligned}
$$

Where, $\boldsymbol{H}$ is an $(m \times G)$ matrix, $G=$ number of PQ buses. Therefore, power flow in line $l$ can be written as

$$
P_{F l}=\left(H_{l}\right)(P)
$$


Where, $\boldsymbol{H}_{l}$ is the $\boldsymbol{I}^{\text {th }}$ row of the matrix $\boldsymbol{H}$. Therefore, power loss in the line $\boldsymbol{l}$ is computed as

$$
\begin{aligned}
L_{l} & =\left(P_{F l}\right)^{2} r_{l}=\left(H_{l} P\right)^{2} r_{l} \\
& =\left(P^{T}\right)\left(H_{l}^{T}\right)\left(H_{l}\right)(P) r_{l} \\
& =\left(P^{T}\right)\left(R^{l}\right)(P)=\sum_{i=1}^{G} \sum_{q=1}^{G} P_{i} R_{i q}^{l} P_{q} \\
& =\sum_{i=1}^{G} P_{i}^{2} R_{i i}^{l}+\sum_{i=1}^{G} \sum_{\substack{q=1 \\
q \neq i}}^{G} P_{i} R_{i q}^{l} P_{q}
\end{aligned}
$$

The coefficient $\left(R^{1}\right)=\left(H_{1}^{T}\right)\left(H_{1}\right) r_{1}$ is a square matrix developed to allocate the loss of line $l$ to the PV buses. Because of this loss coefficient matrix, the procedure accounts for the geographical location of the PV buses in the network.

Assuming that the loss caused by $P_{i}$ and $P_{q}$ is equally allocated to each node of $i$ and $q$. From Eq.17 the allocated loss $L_{G l i}$ to PV bus $i$ for transmission line $l$ can be written as follows:

$$
L_{G l i}=P_{i}^{2} R_{i i}^{1}+\sum_{\substack{\mathrm{q}=1 \\ \mathrm{q} \neq 1}}^{\mathrm{G}} P_{i} R_{i q}^{1} P_{q}
$$

Similarly, loss allocations considering the impact of all other line flows in the network can easily be computed. Therefore, total loss allocated to PV bus $i$ is as

$$
L_{G i}=\sum_{l=1}^{\mathrm{m}} L_{G l i} \ldots \ldots \ldots \ldots \text { (19) }
$$

The sum of the allocated losses to PV buses is exactly equals to the sum of the losses in transmission lines as calculated by using Eq.17. It may be mentioned that the total loss calculated by using Eq.17 is the same as that of calculated by using Eq.8. Also note that sum of the allocated losses to the PQ buses is exactly equals to the sum of the allocated losses to the PV buses.

\section{Algorithm for DC-OPF Using Loss Allocation}

Using above-mentioned procedures for loss allocation a new DC-OPF method has been developed. The proposed algorithm can briefly be written as:

1. Read network data and load level. Set total loss $=0$, penalty factor for ELD mode operation $=1$.

2. Calculate power output (P) of generators by ELD corresponding to total load and total loss.

3. Calculate the percentage of the amount of power injections in generator buses, power flow in transmission lines, total loss, node voltage angles, ITLs and penalty factors.

4. Allocate loss to the load buses and add allocated losses to the respective loads. In every iteration, refresh allocated losses to the loads.

5. Repeat steps 2 through 5 until the network converges and generated power meets total demand. 
6. The percentages of loads including respective losses are recalculated for using them as elements in $(C)$ Eq. 13. Allocate loss to the generator buses.

7. Stop. (End of the algorithm).

This algorithm has been described as follows:

Step 1: Set total loss $L=0$, penalty factor $=1$ etc. The specified load level must be greater than or equal to minimum limits of the generators. Total demand (total load and total loss) must not exceed the sum of upper limits of the generators.

Step 2: Outputs of the generators are calculated in the mode of Economic Load Dispatch by using lambda iteration method which ensures the constraint "total power = total load + total loss”.

Step 3: Applying Eq. 8 and Eq. 14 the total transmission loss, phase angle of every node voltage are obtained and hence, ITLs and penalty factors of the generator buses are calculated. To establish the relationship of power injections in generator buses to those of in load buses, the percentages of power injections (with respect to total generation) in generator buses are also computed by Eq. 2 .

Step 4: Loss allocation is done to the loads. Allocated losses calculated by using Eq. 8, Eq. 9 and Eq.10 are added to the correspon- ding loads. Initially, the matrix $d$ contains only the load in the load demand buses. But in the following iterations $d$ consists of (load + allocated loss) to the respective bus. This allocated loss and the contents of the matrices $C$ and $F$ are repeatedly refreshed until the network converges.

Step 5: The loop iteration continues (repeatedly executes the steps 2 through 5) until generated power meets the total load and total loss.

Step 6: To establish the relationship of power injections in load buses to those of in generator buses, the percentages of power injections in load buses including respective losses allocated to them are computed here. Using Eq. 11 through Eq. 19 loss allocation to generators are calculated properly.

\section{Simulation Results}

To represent the effectiveness of our proposed method, the simulation results have been calculated for the following model power system (Fig.1).

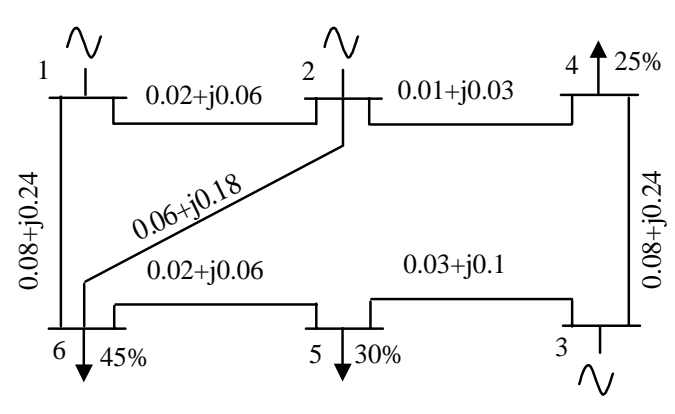

Fig. 1. Model Power System. 
Table I. Characteristic constants of thermal units

\begin{tabular}{|c|c|c|c|c|c|}
\hline \multirow{2}{*}{$\begin{array}{c}\text { Unit } \\
\text { no. }\end{array}$} & \multicolumn{3}{|c|}{ Fuel cost curve constants } & \multicolumn{2}{|c|}{ Output power (MW) } \\
\hline & A & B & C & Min & Max \\
\hline 1 & 15.3 & 1.17 & 0.00145 & 80 & 225 \\
\hline 2 & 13.7 & 1.30 & 0.00163 & 40 & 175 \\
\hline 3 & 10.3 & 1.48 & 0.00226 & 25 & 75 \\
\hline
\end{tabular}

Here, the fuel cost function is $F(P)=$ $\mathrm{A}+\mathrm{BP}+\mathrm{CP}^{2}$.

\section{Loss allocations to the PQ buses (Fig.1)}

Allocated losses to three loads and total losses are shown in Table II. The specified load levels are $150 \mathrm{MW}$ through $400 \mathrm{MW}$. Total losses have been calculated by using Eq. 8 and loss allocations to the loads have been computed by using Eq.9 and Eq.10.
Loss allocations to the PV buses (Fig.1)

Allocated losses to three generators and total losses are shown in Table III. Total losses have been calculated by using Eq.17 and loss allocations to the generators have been computed by using Eq.18 and Eq.19. (Calculation base is 100 MVA). The allocated loss to generator 3 at load level $200 \mathrm{MW}$ is lower than that of $150 \mathrm{MW}$. Though load has been increased from $150 \mathrm{MW}$ to $200 \mathrm{MW}$, the contribution of generator 3 in power flow has comparatively been shrunk (from $16.3 \%$ to $12.18 \%$ of total power). Because the output of Generator 3 remains close to its minimum limit at load level 200 MW. On the other hand, generators 1 and 2 have contributed more power to the network. So, decremented loss allocation to generator 3 at load level 200 MW becomes logically acceptable.

Table II. Allocated losses to the PQ buses

\begin{tabular}{c|c|c|c|c|c|c}
\hline Total Load & $150 \mathrm{MW}$ & 200MW & 250MW & 300MW & 350MW & $400 \mathrm{MW}$ \\
\hline 1 (Bus 4) & 0.2871 & 0.5219 & 0.6859 & 0.8796 & 1.1026 & 1.4963 \\
2 (Bus 5) & 1.2892 & 2.4936 & 3.6447 & 5.0455 & 6.7063 & 9.3376 \\
3 (Bus 6) & 1.7953 & 3.4028 & 5.0548 & 7.0879 & 9.5191 & 13.065 \\
Total Loss & 3.3717 & 6.4182 & 9.3854 & 13.013 & 17.3281 & 23.8989 \\
\hline
\end{tabular}

Table III. Allocated losses to the PV buses

\begin{tabular}{c|c|c|c|c|c|c}
\hline Total Load & 150MW & 200MW & 250MW & 300MW & 350MW & $400 \mathrm{MW}$ \\
\hline Bus 1 & 2.4076 & 4.3636 & 6.0647 & 8.0607 & 10.3587 & 14.2261 \\
Bus 2 & 0.836 & 1.9349 & 2.9754 & 4.2558 & 5.7863 & 8.4531 \\
Bus 3 & 0.1282 & 0.1197 & 0.3452 & 0.6965 & 1.183 & 1.2197 \\
Total Loss & 3.3717 & 6.4182 & 9.3854 & 13.013 & 17.3281 & 23.8989 \\
\hline
\end{tabular}


Table IV shows the allocated losses to the buses for every line loss in the network in Fig. 1 at load level 400 MW. Buses 1, 2 and 3 are PV nodes; buses 4, 5 and 6 are PQ nodes. Total loss has been allocated to the PQ nodes at first and then to the PV nodes. Information about bus to bus connections is shown in the first column in Table IV.
PV bus 1 with about $50 \%$ of the total generation, always gets the highest loss allocated according to all methods. Similarly, all four methods allocate the next highest loss to the PV bus 2 comprising $35 \%$ of the total generation. Similarly, it can be stated that, the PQ bus 6 with $45 \%$ of the system load always gets the highest loss allocated according to all methods.

Table IV. Allocated losses to buses at every line flow

\begin{tabular}{c|c|c|c|c|c|c}
\hline Lines & Bus 1 & Bus 2 & Bus 3 & Bus 4 & Bus 5 & Bus 6 \\
\hline 1 to 2 & 2.2285 & -0.5985 & -0.0424 & 0.7376 & 0.3982 & 0.4518 \\
1 to 6 & 5.9375 & 2.8481 & 0.2019 & 0.451 & 3.1522 & 5.3843 \\
2 to 4 & 0.9635 & 0.8434 & -0.0833 & 1.1149 & 0.3566 & 0.252 \\
2 to 6 & 3.461 & 3.7493 & 0.2657 & -0.4491 & 2.8208 & 5.1044 \\
4 to 3 & 0.6357 & 0.6532 & -0.5792 & -0.3948 & 0.6472 & 0.4573 \\
3 to 5 & 0.8387 & 0.8617 & 1.5936 & 0.0436 & 1.5732 & 1.6772 \\
6 to 5 & 0.1613 & 0.0958 & -0.1366 & -0.0068 & 0.3894 & -0.262 \\
Total loss & 14.2262 & 8.453 & 1.2197 & 1.4963 & 9.3376 & 13.065 \\
\hline
\end{tabular}

\section{Comparison of results of four procedures}

Comparison of the proposed NDC method has been made against a pro-rata (PR) method based on active power, a Proportional Sharing (PS) method based on power flow in the transmission lines and a Marginal procedure based on net power injections and ITLs of the buses. The evaluation of each method can be based on the values of the allocated components measured in MW, in per unit, as a percentage of the system loses. Table $\mathrm{V}$ compares the loss allocation components per bus for different methods. Total loss has been allocated to either PV buses or PQ buses. In this table, the
The highest percentage of loss allocation (calculated by the NDC method) to PV bus 1

Table V. Comparison of the percentages of allocated losses of four procedures for system (Fig.1)

\begin{tabular}{c|c|c|c|c}
\hline Buses & PR & PS & Marginal & NDC \\
\hline PV Bus \#1 & 45.9936 & 44.6659 & 57.2379 & 59.52617 \\
PV Bus \#2 & 36.29007 & 41.6832 & 35.45673 & 35.37025 \\
PV Bus \#3 & 17.71633 & 13.6509 & 7.305364 & 5.10358 \\
Total & 100 & 100 & 100 & 100 \\
& & & & \\
PQ Bus \#4 & 25 & 10.9167 & 10.8740 & 6.26096 \\
PQ Bus \#5 & 30 & 16.8046 & 36.8058 & 39.07125 \\
PQ Bus \#6 & 45 & 72.2788 & 52.32019 & 54.66779 \\
Total & 100 & 100 & 100 & 100 \\
\hline
\end{tabular}


is caused by taking care for the impact of every line flow. The loss coefficient matrices in the NDC method accounts for realizing the impact of line flow, relative positions of the PV and PQ buses in the system. Loss allocations by the NDC method does not depend on ITL of the buses. So, the NDC method is nonvolatile. The PR method actually allocates losses to loads according to the percentages of loads ( $25 \%, 30 \%$ and $45 \%$ of total load) at the PQ buses (see Table V, Column 2). Similarly, loss allocations to the PV buses depend on the percentages of power injections at the respective PV buses. The PR method avoids the network, i.e. it does not take care for the relative positions of the PV and PQ buses in the network. The PS method considers the network by calculating ITLs of the buses. The Marginal procedure is directly ITL based. As a result, both PS and Marginal are highly volatile methods.

\section{Loss allocations in IEEE-118-bus system}

To show the effectiveness of the procedure we have applied it to the IEEE-118-bus system. Table VI shows the allocated losses to the generator and the demand buses at load level 3000 MW. Total loss has been allocated to the demand buses at first and then to the generator buses. It is worth noting that the sum of the allocated losses to the generator buses is exactly equal to the sum of the allocated losses to the demand buses. This procedure can yield negative allocations to reward generators or loads that are strategically well positioned in the network. These negative loss allocations for market purposes can be interpreted as a source of cross-subsidies Conejo, et al. 2003).

Some buses contain both generator and load. As a result, depending on load level, these may be considered either generator buses or load buses. More specifically, if the positive injection from generator is greater than the load of that bus, the bus must be considered as a PV bus. If the positive injection from generator is less than the load of that bus, the bus must be considered as a PQ bus. For example, the 36 generators in the 118-bus system connected to 20 nodes. But, out of them 17 nodes remain as PV nodes and rest 3 nodes become PQ nodes at load level 3000 MW. At 3500 MW load level, 16 nodes remain as PV nodes. Node \#59 becomes PQ node. Similarly, at 4500 MW load level, the nodes \#12, \#59 and \#103 become PQ nodes. Our method also takes care for the transition of PV node to PQ node and vice versa. To reward the geographically well-positioned buses, this procedure can allocate negative losses. For example, negative allocation occurs to buses \#10, \#11, \#12, \#25 and \#26 at load level 3000 MW. But, at load level 4500 MW, negative loss occurs to bus \#103 only. Loss allocation does not depend on whether the ITL of that bus is negative or positive. So, this method is non-volatile. The NDC method does not allocate loss to the bus that has neither load nor generator. 
Table VI. Allocated losses to the loads and generators for the 118-Bus system

\begin{tabular}{c|c|c|c|c|c|c|c|c|c|c|c}
\hline $\begin{array}{c}\text { Gen. } \\
\text { Buses }\end{array}$ & Loss & $\begin{array}{c}\text { Load } \\
\text { buses }\end{array}$ & Loss & $\begin{array}{c}\text { Load } \\
\text { buses }\end{array}$ & Loss & $\begin{array}{c}\text { Load } \\
\text { buses }\end{array}$ & Loss & $\begin{array}{c}\text { Load } \\
\text { buses }\end{array}$ & $\begin{array}{c}\text { Loss } \\
\text { boad } \\
\text { buses }\end{array}$ & Loss \\
\hline 10 & -0.10987 & 1 & 0.21691 & 28 & 0.08274 & 50 & 0.06028 & 79 & 0.10519 & 105 & 0.07939 \\
11 & -0.24090 & 2 & 0.19025 & 29 & 0.19153 & 51 & 0.12727 & 82 & 0.05489 & 106 & 0.06229 \\
12 & -0.02009 & 3 & 0.20741 & 31 & 0.16529 & 52 & 0.14333 & 83 & 0.06891 & 107 & 0.11270 \\
25 & -0.12518 & 4 & 0.17534 & 32 & 0.19383 & 53 & 0.15882 & 84 & 0.10941 & 108 & 0.01865 \\
26 & -0.03476 & 6 & 0.19177 & 33 & 0.09898 & 54 & 0.26716 & 85 & 0.06082 & 109 & 0.01868 \\
49 & 0.94221 & 7 & 0.17513 & 34 & 0.09256 & 55 & 0.36206 & 86 & 0.15073 & 110 & 0.08864 \\
59 & 0.01524 & 13 & 0.17256 & 35 & 0.10899 & 56 & 0.31622 & 88 & -0.04170 & 112 & 0.07305 \\
61 & 0.16279 & 14 & 0.16034 & 36 & 0.06815 & 57 & 0.27709 & 90 & -0.10892 & 114 & 0.03991 \\
65 & 1.22737 & 15 & 0.12503 & 39 & 0.15085 & 58 & 0.29484 & 92 & -0.01529 & 115 & 0.12008 \\
66 & 1.09458 & 16 & 0.09519 & 40 & 0.14721 & 60 & 0.14284 & 93 & -0.00273 & 117 & 0.22276 \\
69 & 1.67753 & 17 & 0.03300 & 41 & 0.16203 & 62 & 0.11945 & 94 & 0.00370 & 118 & 0.23941 \\
80 & 1.45308 & 18 & 0.14208 & 42 & 0.11678 & 67 & 0.06311 & 95 & 0.00554 & Total & 9.18607 \\
& & & & & & & & & & loss & \\
87 & 0.00346 & 19 & 0.12675 & 43 & 0.05418 & 70 & 0.14107 & 96 & 0.01533 & & \\
89 & 3.06362 & 20 & 0.13557 & 44 & 0.04707 & 74 & 0.20341 & 97 & 0.00362 & & \\
100 & 0.03265 & 21 & 0.07643 & 45 & 0.12351 & 75 & 0.15789 & 98 & 0.00493 & & \\
103 & 0.01263 & 22 & 0.07218 & 46 & 0.07646 & 76 & 0.17044 & 101 & 0.00425 & & \\
111 & 0.03168 & 23 & 0.03011 & 47 & 0.03893 & 77 & 0.06250 & 102 & -0.00950 & & \\
Total & 9.18607 & 27 & 0.17555 & 48 & 0.03732 & 78 & 0.11225 & 104 & 0.06329 & & \\
loss & & & & & & & & & & &
\end{tabular}

\section{A. Special case}

If a bus contains both load and generator, the ITL of that bus cannot give forth the true figure of penalty factor. As a result, at this PV bus, true figure of net power injection does not occur. Therefore, power output on the basis of penalty factor, in view of ELD approach, becomes unexpectedly incorrect. It comes from the fact that the generator's output is governed by the inaccurate ITL that depends on net positive power injection. To overcome this drawback (Eq. 4) has been used faithfully to determine the power injections in the PV buses. Anyway, where ELD approach has been disregarded, the percentages of power outputs in the matrix $F$ Eq. 3 should be computed from the net positive power injections in the generator buses.

\section{Conclusions}

A new transmission loss allocation procedure and its application to DC-OPF has been 
proposed and tested. It can be concluded that the procedure is based on the exact network equations in DC load flow method. The system losses are shown to be separable among the buses naturally because of the loss coefficient matrices. The loss coefficient matrices derived from transmission line loss calculation equation are useful tools in the loss allocation equations (one for loss allocations to loads and the other for loss allocations to generators). It is extremely simple to formulate and to implement. In a similar way to incremental loss allocation methods, this procedure can yield negative loss allocation to reward geographically well-positioned generators or loads in the network. Another property of the suggested method is that it does not require the choice of an arbitrary slack bus.

Using our procedure the total loss can be allocated to the loads and/or to the generators. The loss coefficient matrices $S$ and $R$ developed in our procedure play a vital rule for loss allocation to loads and generators respectively. By using $S$ and $R$ matrices the loss allocation formulas consider the impact of every line flow in the network. The outstanding property of our procedure is the development of the loss coefficient matrices ( $S$ and $R$ ) that have explicitly taken account the respective positions of the buses.

\section{References}

Conejo, A.J. Galiana, F.D. Arroyo, J.M. GarciaBertrand, R. Chua, C.W. and Huneault, M. (2003) Economic inefficiencies and crosssubsidies in an auction-based electricity pool, IEEE Trans. on Power Syst., 18(1) : 211-228.

Conejo, A.J. Arroyo, J.M. Alguacil, N. and Guijarro, A.L. (2002) Transmission loss allocation: a comparison of different practical algorithms, IEEE Trans. on Power Syst., 17(3) : 571-576.

Conejo, A.J. Galiana, F.D. and Kochar, I. (2001) Z-bus loss allocation, IEEE Trans. on Power Syst., 16(1) : 105-110.

Exposito, A.G. Santos, J.M.R. Garcia, T.G. and Velasco, E.A.R. (2000). Fair Allocation of Transmission Power Losses, IEEE transactions on Power Systems, 15(1) : 184-188.

Galiana, F.D. Conejo, A.J. and Gil, H.A. (2003) Transmission network cost allocation based on equivalent bilateral exchanges, IEEE Trans. on Power Syst., 18(4) : 1425-1431.

Galiana, F.D. Conejo, A.J. and Kochar, I. (2002) Incremental transmission loss allocation under pool dispatch, IEEE Trans. on Power Syst., 17(1) : 26-33.

Gross, G. and Tao, S. (2000) A physical-flowbased approach to allocating transmission 
losses in a transaction framework, IEEE Trans. on Power Syst., 15(2) : 631-637.

Kirschen, D. Allan, R. and Strbac, G. (1997) Contributions of individual generators to loads and flows, IEEE Trans. Power Syst., 12 : 52-60.

Mateus, J.C. and Franco, P.C. (2005). Transmission loss allocation through equivalent bilateral exchanges and economic analysis, IEEE Trans. on Power Syst., 20(4) : 1799-1807.
Wood A.J. and Wollenberg, B.F. (1996) Power Generation, Operation and Control. Second edition, New York: John Wiley \& Sons,. Ch. 3.

Yamashiro, S. (1977) Optimization of power flow by DC method, IEEJ Trans. PE, 97B(11) : 679-684.

Received : March 11, 2007;

Accepted : August 15, 2007 